\title{
Pro/con debate
}

\section{Con: Access to advanced}

\section{therapies for severe asthma} should be restricted to patients

\section{with satisfactory adherence to maintenance treatment}

Current guidance states that advanced therapies should only be used when adherence to maintenance therapy (inhaled corticosteroid/long-acting $\beta$-agonist) has been proven. This is based on the costs of advanced therapies, the fact that they were generally trialled as add-ons to maintenance therapy, and the assumed efficacy of maintenance therapy in the majority of adherent patients.

In this pro/con debate, we argue that such a rigid view of access downplays the complex and multifactorial nature of poor adherence. Not only does the evidence indicate a role for psychosocial factors in both poor adherence and poor asthma outcomes, failure of maintenance therapy itself may be a driver of poor adherence behaviours. Some individuals at high risk of poor asthma outcomes will therefore also have poor adherence that is not rapidly amenable to intervention. Rather than punishing them for factors outside of their control, they should be allowed access to advanced therapies in order to reduce their adverse risk resulting from uncontrolled asthma.

\section{Introduction}

The heart of this debate is about money and who deserves a piece of the pie, more politely described as "resource allocation". The advanced therapies discussed here are injectable monoclonal antibody (biologic) treatments. They are undoubtedly expensive, and it is often argued that their unrestricted use is not cost-effective. Studies showing their efficacy have largely, to date, examined their use as an add-on treatment rather than monotherapy. Consequently, patients are required to demonstrate good adherence to maintenance treatment prior to being offered access to biologics. This is reflected in current guidelines [1].

This article will argue that cost lies at the heart of this debate. It will argue that poor inhaled multifactorial and will rarely resolve with a single outpatient visit. It will argue that restriction of advanced therapies based on cost considerations is short-sighted as the stakes of under-treatment in uncontrolled asthma are high. It will finally argue that, rather than being shoehorned into the role of gatekeepers, clinicians must be trusted to use the tools at their disposal to seek the best outcomes for all of their patients. corticosteroid (ICS) adherence is complex,

@ERSpublications

The restriction of access to monoclonal antibody therapies based on adherence in severe asthma is both short-sighted and a false economy. It fails to recognise that poor adherence is complex, multifactorial and resistant to intervention. https://bit.ly/3uDCCL7
Cite as: Adejumo I, Shaw DE. Con: Access to advanced therapies for severe asthma should be restricted to patients with satisfactory adherence to maintenance treatment. Breathe 2021; 17: 210049 . 


\section{A question of money}

No head-to-head trials have compared ICS (the standard of care in asthma) with biologics. It is likely that these would have been conducted to assess a potential role for biologics as first-line therapies had biologics been cheaper than ICS inhalers. There is furthermore an indication that benefit from biologics may not depend on continued adherence to high-dose maintenance therapy. Data from WENZEL et al. [2] suggested dupilimab efficacy continued following withdrawal of longacting $\beta$-agonist (LABA) and subsequent tapering and withdrawal of ICS. In a retrospective analysis of patients commenced on benralizumab, D'ANCONA et al. [3] demonstrated that poor and intermediate ICS adherence were not associated with worse outcomes when compared with individuals with good ICS adherence. Arguments to restrict use of biologics must therefore be centred on cost. It is understandable that commissioners fear a tsunami of patients overwhelming services. Such fear is misplaced.

The prevalence of "difficult-to-control" asthma has been estimated at $17 \%$ of adults with asthma, with half of this group taking $<80 \%$ of their prescribed inhaler doses [4]. A small subset, possibly between 24 and 35\% [5], of these individuals will be eligible to trial a biologic, dependent on their baseline treatment step, biomarker status, lung function and historical systemic corticosteroid requirements. This is no tsunami. When the total societal and healthcare utilisation costs due to asthma exacerbations and side-effects of regular or recurrent systemic glucocorticoid use are taken into consideration [6], it may barely even prove to be a financial ripple.

\section{Poor adherence is complex and multifactorial}

Poor adherence in the presence of uncontrolled asthma is neither an arbitrary phenomenon nor a lifestyle choice. Like many systems, the medical establishment is quick to blame the victim and slow to understand the context. The causes of poor adherence are complex and multifactorial, and it is beyond the scope of this article to detail them all; however, three key issues are examined below.

\section{The drugs don't work}

The nature of scientific reporting generally places the emphasis on group mean effects, with presentation of individual responses often relegated to the data supplement. MALMSTrom et al. [7] elegantly demonstrate how a significant proportion of individuals within the group may experience effects which are incongruent with the overall group effect. In their study, lung function improved in response to both montelukast and beclometasone. However, in a third of participants from the montelukast arm and a quarter of participants from the beclometasone arm there was a deterioration in lung function. In the real world, despite evidence of population-level effectiveness, poor individual response may lead to poor adherence.

\section{It's complicated}

Guidelines in the UK suggest almost 100 different ICS and combination inhaler maintenance regimes using over 30 devices [1]. This begs the question why so many different devices and formulations if this regime works so well? By the time a patient is referred to a severe asthma centre, they are likely to have tried a multiplicity of coloured inhalers, all used slightly differently. Patients may be supplied with a dry powder ICS/LABA (breathe in hard and fast, do not shake device) and a reliever metered dose inhaler (breathe in slowly and deeply, shake device before use) simultaneously. Healthcare professionals themselves have a poor understanding of inhaler technique [8]. It is therefore unsurprising that poor inhaler technique is common and is itself linked to poor outcomes [9]. Thus, ineffective dosing due to poor technique leading to poor treatment response may result in poor adherence.

\section{Psychological and social determinants}

Poor adherence has been linked to social disadvantage and psychological comorbidity [10]. Rather than a paternalistic "try harder" approach, more effective adherence interventions may include free prescriptions (where this is not already the case), free public transport, or more accessible pharmacies that are open outside of working hours. Some may need help with chaotic personal circumstances, provision of carer support or treatment for psychiatric comorbidities. While asthma services can play a role in addressing some of these factors (incorporating psychological services, for example), a holistic approach is required involving input from other services including primary care, mental health services and social care.

Poor asthma control itself has strong links to societal disadvantage [11]. That societal disadvantage is a common factor for both poor adherence and poor control is unlikely to be a coincidence. More likely, poor control contributes to a complex matrix in which poor adherence thrives through psychological comorbidity and loss of productivity. This becomes a vicious cycle, not helped by simply telling patients to take their inhalers.

The association between health inequality and societal disadvantage is not unique to asthma and has been the subject of national and international 
commissions [12]. In cardiovascular disease, poverty is implicated in baseline risk factors including hypertension and diabetes, and environmental factors such as community health behaviour and access to infrastructure such as parks and sports facilities [12]. In diabetes, poverty increases the likelihood of poor glycaemic control and microand macrovascular complications [12]. However, patients are still offered percutaneous coronary angiograms even if they haven't been taking their aspirin, and expensive flash glucose monitoring systems are actively encouraged in patients with Type 1 diabetes and difficult psychosocial circumstances.

To restrict access to biologics on the basis of poor adherence singularly positions respiratory medicine as a specialty which widens preexisting health and societal inequalities. Such an approach would be in stark contrast to other specialties in addition to those already mentioned, such as psychiatry and infectious diseases, where the availability of clinician-administered depot injections or directly observed therapy ensures that the most vulnerable and at-risk patients are still treated, particularly when there is deemed to be a risk of poor adherence.

\section{No easy fix}

If poor adherence is multifactorial and complex, it follows that its solutions are likely to be also. Crucially, a 2017 Cochrane review of adherence interventions noted that interventions do not consistently translate into improved clinical outcomes [13]. Adherence studies suggest that some individuals display non-adherence that is resistant to educational interventions [14]. There is therefore a serious risk that by conducting potentially futile and poorly evidenced adherence experiments, effective and evidence-based treatment (i.e. monoclonal antibody therapy) is withheld. In the meantime, the individual deteriorates faster than the complex reasons behind their non-adherence can be addressed. Despite all this, clinicians still emphasise the need to optimise adherence prior to escalating to a potentially lifesaving biologic.

\section{The stakes are high}

Poor ICS adherence is not benign. In the UK, a confidential enquiry into asthma deaths (National Review of Asthma Deaths; NRAD) found it to be an avoidable factor in a quarter of those who had died [15]. Although deaths in asthma are rare events and falling over time [16], complacency is clearly costly.

Studies show that individuals with severe asthma use more medications, experience more hospitalisations and are more likely to experience loss of productivity when compared with individuals with mild or moderate asthma [6]. Their poorly controlled symptoms and frequent asthma attacks lead to an increased prevalence of anxiety and depression as well as a reduced quality of life [17], with psychological comorbidity in particular implicated in asthma deaths [15].

Until recently, the primary mode of add-on treatment available to this subgroup was regular oral corticosteroid (OCS) therapy, which comes with a heavy side-effect profile. An increased risk of complications, including cataracts, peptic ulcer disease, sepsis, fractures, metabolic conditions, cardiovascular risk, sleep apnoea, and psychological comorbidity, may occur with the equivalent of only four acute courses over a lifetime [18], placing significant additional burden on individuals already suffering the adverse effects of severe asthma. They are also associated with increased healthcare costs [6].

Advanced therapies are costly but work, reducing asthma attacks and hospital admissions with a minimal side-effect profile [19]. There is a financial cost to withholding their use, with costs of refractory asthma in the UK estimated to be up to GBP 4000 per year in direct costs, not accounting for societal costs. What other area of healthcare practice denies a patient access to a treatment that works because of non-adherence to a treatment that doesn't?

\section{A pragmatic approach}

This is not the first time that an alternative approach has been suggested. Following the tragic death of a teenager who was deemed ineligible for access to biologic therapies due to a history of poor adherence, GREEN et al. [20] made the case for use of a short course of monoclonal antibody therapy to buy time for adherence interventions. Severe asthma services should be identifying highrisk patients with resistant poor adherence and uncontrolled disease (particularly where adherence has been complicated by poverty, social exclusion or mental health issues) early in their patient journey. These individuals should be provided with additional support (e.g. access to psychological services) and considered a priority for access to biologics which may, in providing effective treatment, help them better engage in their care. Restrictions exist to allow healthcare systems with limited resources to provide these treatments to the individuals who need them most. Patients with complex poor adherence are part of that group. Access for them may be lifesaving.

\section{Conclusion}

Uncontrolled asthma has potentially serious effects on morbidity, mortality, psychological health and 
socioeconomic circumstances. Poor medication adherence does the same. This pro/con debate offers two visions. Clinicians and commissioners may choose to position themselves based on a concern for cost. The result of such a position may be poorer outcomes, widening health inequalities and, ironically, higher costs.

We argue for a vision of advanced therapies where standard ICS therapy has failed for high-risk, but potentially high reward, individuals.

\section{Affiliations}

\section{Ireti Adejumo, Dominick E. Shaw}

Respiratory Medicine, NIHR Nottingham Biomedical Research Centre, Nottingham, UK.

\section{Conflict of interest}

I. Adejumo reports PhD studentship funding from GlaxoSmithKline for an investigator-led grant paid to me via my institution 2016-2019. D.E. Shaw reports support for attending meetings/travel from AstraZeneca and TEVA outside the submitted work.

\section{References}

1. British Thoracic Society, Scottish Intercollegiate Guidelines Network. British guideline on the management of asthma: A national clinical guideline. 2019. www.brit-thoracic.org.uk/ quality-improvement/guidelines/asthma/ Date last updated: July 2019; date last accessed: 17 March 2021.

2. Wenzel S, Ford L, Pearlman D, et al. Dupilumab in persistent asthma with elevated eosinophil levels. N Engl J Med 2013 368: 2455-2466.

3. d'Ancona G, Kavanagh JE, Dhariwal J, et al. Adherence to inhaled corticosteroids and clinical outcomes following a yea of benralizumab therapy for severe eosinophilic asthma. Allergy 2021; in press [https://doi.org/10.1111/all.14737].

4. Hekking P-PW, Wener RR, Amelink M, et al. The prevalence of severe refractory asthma. J Allergy Clin Immunol 2015; 135 896-902.

5. Albers FC, Müllerová H, Gunsoy NB, et al. Biologic treatment eligibility for real-world patients with severe asthma: The IDEAL study. J Asthma 2018; 55: 152-160.

6. O'Neill S, Sweeney J, Patterson CC, et al. The cost of treating severe refractory asthma in the UK: an economic analysis from the British Thoracic Society Difficult Asthma Registry. Thorax 2015; 70: 376-378.

7. Malmstrom K, Rodriguez-Gomez G, Guerra J, et al. Oral montelukast, inhaled beclomethasone, and placebo for chronic asthma. A randomized, controlled trial. Montelukast/ Beclomethasone Study Group. Ann Intern Med 1999; 130 487-495.

8. Plaza V, Giner J, Rodrigo GJ, et al. Errors in the use of inhalers by health care professionals: a systematic review. J Allergy Clin Immunol Pract. 2018; 6: 987-995.

9. Price DB, Roman-Rodriguez M, McQueen RB, et al. Inhale errors in the CRITIKAL study: type, frequency, and association with asthma outcomes. J Allergy Clin Immunol Pract 2017; 5: 1071-1081.

10. Apter AJ, Boston RC, George M, et al. Modifiable barriers to adherence to inhaled steroids among adults with asthma: it's not just black and white. J Allergy Clin Immunol 2003; 111 : 1219-1226.

11. Forno E, Celedón JC. Health disparities in asthma. Am J Respir Crit Care Med 2012; 185: 1033-1035.

12. Blas E, Sivasankara Kurup A. Equity, Social Determinants and Public Health Programmes. Geneva, World Health Organisation, 2010. https://apps.who.int/iris/handle/106 $65 / 44289$

13. Normansell R, Kew KM, Stovold E. Interventions to improve adherence to inhaled steroids for asthma. Cochrane Database Syst Rev 2017; 4: CD012226.

14. Sulaiman I, Greene G, MacHale E, et al. A randomised clinical trial of feedback on inhaler adherence and technique in patients with severe uncontrolled asthma. Eur Respir J 2018; 51: 1701126.

15. Levy ML, Andrews $\mathrm{R}$, Buckingham $\mathrm{R}$, et al. Why asthma still kills: The National Review of Asthma Deaths (NRAD). Confidential enquiry report. London, Royal College of Physicians, 2014. www.rcplondon.ac.uk/projects/outputs/ why-asthma-still-kills

16. Global Asthma Network. The Global Asthma Report 2018. Auckland, Global Asthma Network, 2018. http:// globalasthmareport.org/

17. Shaw DE, Sousa AR, Fowler SJ, et al. Clinical and inflammatory characteristics of the European U-BIOPRED adult severe asthma cohort. Eur Respir J 2015; 46: 1308-1321.

18. Price DB, Trudo F, Voorham J, et al. Adverse outcomes from initiation of systemic corticosteroids for asthma: long-term observational study. J Asthma Allergy 2018; 11: 193-204.

19. Farne HA, Wilson A, Powell C, et al. Anti-IL5 therapies for asthma. Cochrane Database Syst Rev 2017; 9: CD010834.

20. Green RH, Shaw D. Strict adherence rules to obtain monoclonal therapy might cost lives. Lancet Respir Med 2017, 5. 678-679 